\title{
In vivo functional assessment of a novel degradable metal and elastomeric scaffold-based tissue engineered heart valve
}

\author{
Garrett N. Coyan, MD, MS, ${ }^{\mathrm{a}, \mathrm{b}}$ Antonio D’Amore, PhD, ${ }^{\mathrm{a}, \mathrm{b}, \mathrm{c}}$ Yasumoto Matsumura, MD, ${ }^{\mathrm{a}, \mathrm{b}}$
}

Drake D. Pedersen, BS, ${ }^{\mathrm{a}, \mathrm{b}}$ Samuel K. Luketich, BS, ${ }^{\mathrm{a}, \mathrm{b}}$ Vesselin Shanov, PhD, ${ }^{\mathrm{d}}$ William E. Katz, MD,

Tirone E. David, MD, ${ }^{f}$ William R. Wagner, $\mathrm{PhD},{ }^{\mathrm{a}, \mathrm{b}}$ and Vinay Badhwar, $\mathrm{MD}^{\mathrm{b}, \mathrm{g}}$

\section{ABSTRACT}

Objective: Ideal heart valve solutions aim to provide thrombosis-free durability. A scaffold-based polycarbonate urethane urea tissue-engineered heart valve designed to mimic native valve microstructure and function was used. This study examined the acute in vivo function of a stented tissue-engineered heart valve in a porcine model.

Methods: Trileaflet valves were fabricated by electrospinning polycarbonate urethane urea using double component fiber deposition. The tissue-engineered heart valve was mounted on an AZ31 magnesium alloy biodegradable stent frame. Five 80-kg Yorkshire pigs underwent open tissue-engineered heart valve implantation on cardiopulmonary bypass in the pulmonary position. Tissue-engineered heart valve function was echocardiographically evaluated immediately postimplant and at planned study end points at 1, 4, 8, and 12 hours. Explanted valves underwent biaxial mechanical testing and scanning electron microscopy for ultrastructural analysis and thrombosis detection.

Results: All 5 animals underwent successful valve implantation. All were weaned from cardiopulmonary bypass, closed, and recovered until harvest study end point except 1 animal that was found to have congenital tricuspid valve dysplasia and that was euthanized postimplant. All 5 cases revealed postcardiopulmonary bypass normal leaflet function, no regurgitation, and an average peak velocity of $2 \mathrm{~m} / \mathrm{s}$, unchanged at end point. All tissue-engineered heart valve leaflets retained microstructural architecture with no platelet activation or thrombosis by scanning electron microscopy. There was microscopic evidence of fibrin deposition on 2 of 5 stent frames, not on the tissueengineered heart valve. Biaxial stress examination revealed retained postimplant mechanics of tissue-engineered heart valve fibers without functional or ultrastructural degradation.

Conclusions: A biodegradable elastomeric heart valve scaffold for in situ tissueengineered leaflet replacement is acutely functional and devoid of leaflet microthrombosis. (J Thorac Cardiovasc Surg 2019;157:1809-16)

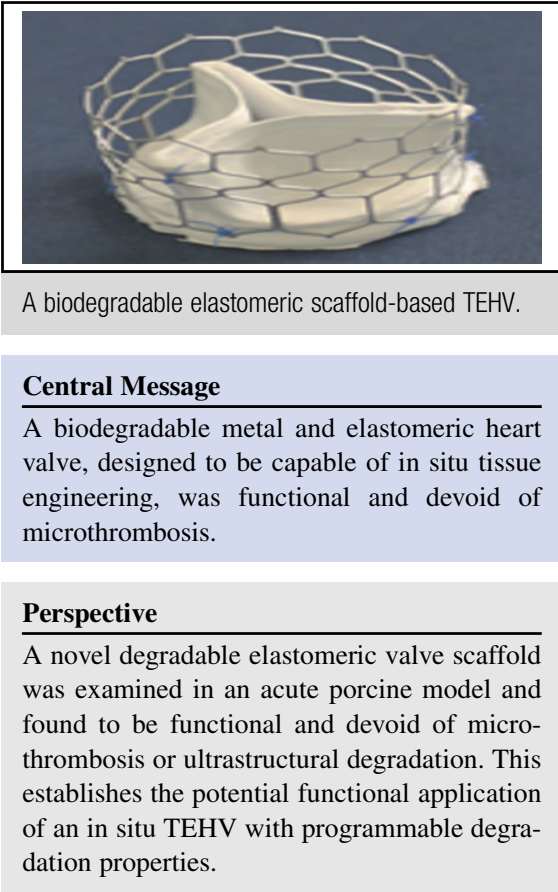

See Commentary page 1817.

\footnotetext{
From the ${ }^{a}$ Department of Cardiothoracic Surgery, University of Pittsburgh, Pittsburgh, Pa; ${ }^{\mathrm{b}} \mathrm{McG}$ owan Institute for Regenerative Medicine, Pittsburgh, Pa; ${ }^{\mathrm{c}}$ Fondazione RiMED, Palermo, Italy; ${ }^{\mathrm{d}}$ Department of Bioengineering, University of Cincinnati, Cincinnati, Ohio; ${ }^{e}$ Division of Cardiology, University of Pittsburgh, Pittsburgh, Pa; ${ }^{\mathrm{f}}$ Division of Cardiac Surgery, Toronto General Hospital, Toronto, Ontario, Canada; and ${ }^{\mathrm{g}}$ Department of Cardiovascular and Thoracic Surgery, West Virginia University, Morgantown, WVa.

This work was supported by the Wallace H. Coulter Foundation Translational Bioengineering Research Award, the Clinical and Translational Science Institute,University of Pittsburgh, and the RiMED Foundation (Grant 0057091).
}

Read at the 98th Annual Meeting of The American Association for Thoracic Surgery, San Diego, California, April 28-May 1, 2018.

Received for publication April 8, 2018; revisions received Sept 1, 2018; accepted for publication Sept 22, 2018; available ahead of print Dec 18, 2018.

Address for reprints: Vinay Badhwar, MD, Department of Cardiovascular and Thoracic Surgery, 1 Medical Center Drive, Morgantown, WV 26506 (E-mail: vinay.badhwar@wvumedicine.org).

$0022-5223 / \$ 36.00$

Copyright (C) 2018 by The American Association for Thoracic Surgery

https://doi.org/10.1016/j.jtcvs.2018.09.128 


\section{Abbreviations and Acronyms \\ DCD = double-component deposition \\ $\mathrm{Mg}=$ magnesium \\ PCUU $=$ polycarbonate urethane urea \\ SEM = scanning electron microscopy \\ $\mathrm{TEHV}=$ tissue-engineered heart valve}

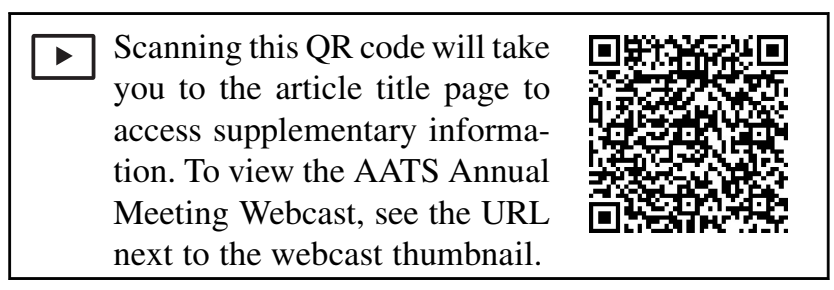

Lifesaving and successful prosthetic heart valve replacement operations have inherent limitations. Mechanical valve durability is accompanied by liabilities associated with lifelong anticoagulation. Bioprostheses afford patients the opportunity to avoid rigorous anticoagulation in exchange for limited long-term durability secondary to wellrecognized modes of failure. ${ }^{1-3}$

Valvular heart disease remains a global management challenge of access to optimal therapy, especially in children and in developing countries where rheumatic disease remains prevalent. ${ }^{4}$ The ideal valve prosthesis provides for nonthrombogenic long-term durability and the potential to adapt to somatic growth. A potential solution is a tissueengineered heart valve (TEHV).

Techniques for TEHV development have included in vitro fabrication, cell seeding, conditioning and secondary implantation, and, more recently, in situ scaffold implantation and subsequent autologous tissue replacement. ${ }^{5,6}$ The former has been challenged by functional tissue replication adaptive to a dynamic hemodynamic environment, and the latter by acute durability and effective subsequent cellular resurfacing with scaffold degradation. An elastomeric, degradable polymeric scaffold ${ }^{7,8}$ was generated in the form of a functional trileaflet heart valve from polycarbonate urethane urea (PCUU) using a novel electrospinning process and tested by cyclical pulse duplication. ${ }^{9}$

The goal of this study was to examine the microstructural integrity, hemodynamic function, and biocompatibility of the PCUU trileaflet heart valve mounted on a biodegradable magnesium $(\mathrm{Mg})$ stent frame surgically implanted in the pulmonary position in an acute porcine model. The applications of a fully biodegradable stented TEHV extended to all valve positions with the potential of forming a completely bioreplaceable valve over time may have broad appeal as a disruptive technology to address the clinical and knowledge gap in search of a potentially nonthrombogenic and durable valve solution. ${ }^{1,4-6}$

\section{MATERIALS AND METHODS \\ Ethics Statement}

The study protocol was approved by the Institutional Animal Care and Use Committee of the University of Pittsburgh. Animals were cared for in accordance with the National Institutes of Health Guide for the Care and Use of Laboratory Animals.

\section{Fabrication of Valve Scaffold and Stented Valve Fabrication}

Scaffold-based PCUU valves were fabricated and processed with a double-component deposition (DCD) technique and electrospun on a semilunar valve mandrel. ${ }^{9}$ The following electrospinning conditions were used: voltage gap $13 \mathrm{kV}$, gap $4.5 \mathrm{~cm}$, rotational speed $200 \mathrm{rpm}$, polymer flow rate $1.5 \mathrm{~mL} / \mathrm{h}$, and mandrel major diameter $23 \mathrm{~mm}$. Mg AZ31 alloy was processed using photochemical etching to create a stent that was biodegradable over time. ${ }^{10,11}$ The scaffold-based PCUU valve was mounted on the $\mathrm{Mg}$ stent using 5-0 polypropylene sutures distributed at 6 locations as illustrated (Figure 1). The entire prosthesis was then sterilized by 3 serial $70 \%$ ethanol washes for 20 minutes, followed by 3 washes in phosphate-buffered saline for 20 minutes. The process was conducted inside a biological hood where each device was cumulatively exposed to ultraviolet light.

\section{Surgical Procedure}

Five Yorkshire pigs were obtained and underwent preoperative veterinary assessment and acclimatization before surgical procedure. Anesthesia was induced with intramuscular ketamine, inhaled isoflurane, and intramuscular Telazol. General anesthesia was maintained throughout the operation with titration of isoflurane by a veterinary anesthesia team. Animals were intubated, and subsequently internal jugular venous cannulation and carotid arterial cannulation were completed via direct cutdown for monitoring and venous access. Median sternotomy was performed, and normothermic cardiopulmonary bypass was established via bicaval venous cannulation and ascending aortic cannulation after complete systemic heparinization. Adequate heparin dosing was gauged by activated clotting time of greater than 410 seconds.

The pulmonary artery was incised directly during beating-heart cardiopulmonary bypass, and the native pulmonary valve was excised. The stented PCUU prosthesis was implanted using a running 4-0 polypropylene annular suture. The pulmonary artery was closed, cardiopulmonary bypass was weaned, and the animal was decannulated followed by protamine sulfate administration. Activated clotting time was obtained after protamine administration to ensure return to baseline coagulation parameters. Epicardial 2-dimensional echocardiography was completed intraoperatively and at planned study end points of 1, 4, 8, and 12 hours. Animals were continuously monitored by veterinary intensive care staff for 1 to 12 hours after valve implant per study protocol, and vital signs and serial arterial blood gas measurements were used during the postoperative period. The initial animal was extubated, with the remaining animals being ventilated throughout the postoperative study period prophylactically to prevent isolated noncardiac respiratory complications that had developed in the first animal. Animals underwent euthanasia at the determined study end point or if a humane end point was reached according to study protocol.

\section{Prosthetic Implant Analysis}

All animals underwent postoperative epicardial echocardiography to assess acute valve function after surgical implantation. Echocardiographic evaluation included valve leaflet motion and prosthetic valve positioning, 


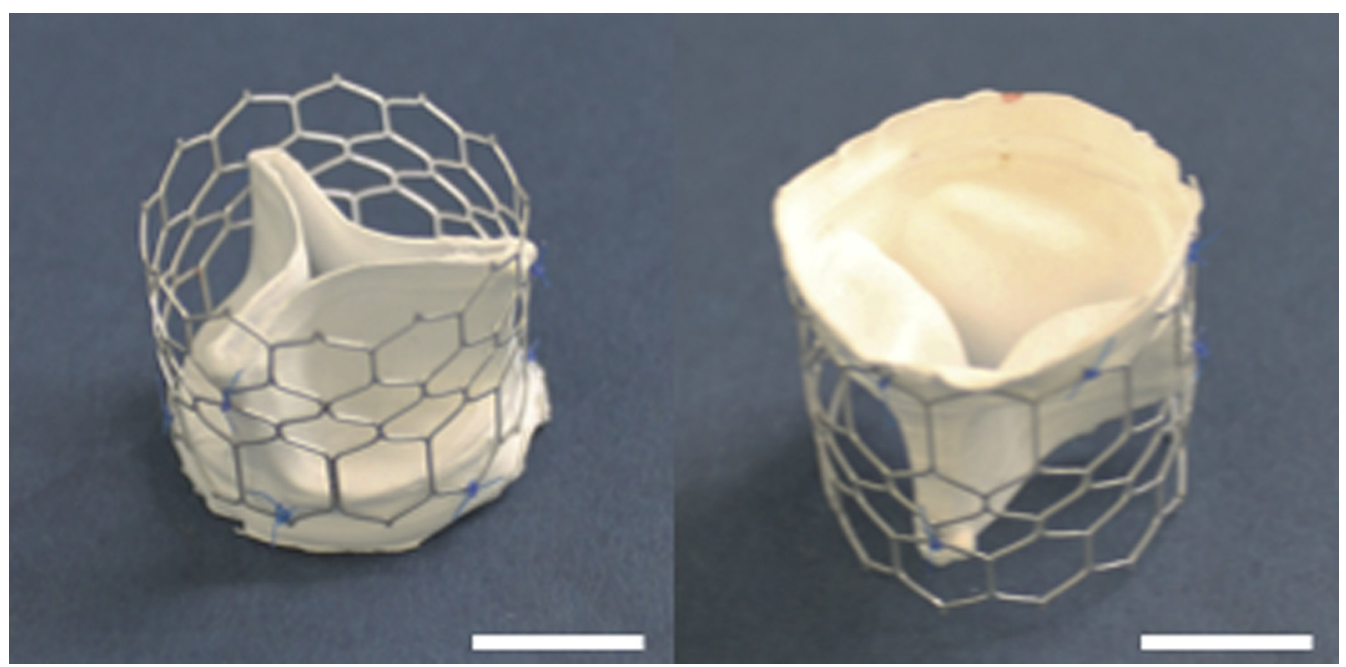

FIGURE 1. PCUU pulmonary valve fabricated with DCD electrospinning mounted on an Mg AZ 31 alloy degradable metallic stent.

continuous-wave Doppler to quantify forward flow through the TEHV and any potential regurgitation, and color flow Doppler to identify any turbulence or regurgitant jet of the TEHV. Epicardial echocardiography was also performed before study end point/euthanasia in 4 animals occurring at 1-, 4-, 8-, and 12-hour time points to confirm valve function was unchanged.

Gross explant analysis was completed immediately after study end point occurred. Implants were assessed for gross degradation or damage, thrombosis, or any other malfunction. The 5 PCUU valves were carefully removed from the $\mathrm{Mg}$ alloy stents, and all underwent separate analysis. One PCUU leaflet from each valve was delegated for biaxial mechanical testing, one for histologic evaluation of early cellular infiltration, and one for scanning electron microscopy (SEM) for surface analysis. The $5 \mathrm{Mg}$ stents also underwent SEM analysis to examine surface morphology.

Biaxial stress-strain analysis in stress control mode was performed using a customized biaxial tensile testing device. ${ }^{9}$ Histology was performed by fixing samples in $10 \%$ formalin for fixation and sectioning, followed by hematoxylin-eosin staining to identify cells within the PCUU valve scaffold cross-section. SEM imaging was performed on PCUU leaflets after fixation in $2 \%$ glutaraldehyde and $\mathrm{Mg}$ stent samples after sputter coating with Pd/Au on a standard SEM (Jeol JSM633OF).

\section{Statistical Methods}

Numeric data are presented as mean \pm standard deviation. There were no comparisons or advanced statistical methods used in this pilot study because of limited sample size.

\section{RESULTS}

\section{Surgical Tissue-Engineered Heart Valve Implant Procedure}

Five Yorkshire pigs, weight $89 \pm 3 \mathrm{~kg}$, underwent successful TEHV placement in the described manner (Figure 2). The PCUU sewing cuff performed well and held the polypropylene suture well. The Mg AZ31 stent body was rigid and brittle, with deformation occurring under normal surgical handling conditions. The first animal was found to have a large atrial septal defect with tricuspid valve dysplasia and was unable to be weaned from cardiopulmonary bypass after TEHV placement. The 4 subsequent animals were successfully weaned from cardiopulmonary bypass and had the chest closed. Protamine administration normalized the activated clotting time to baseline postoperatively in all subjects. Mean cardiopulmonary bypass time was $92 \pm 19$ minutes. Of note, each successive case had a shorter cardiopulmonary bypass time as the model developed, with the final case being 64 minutes.

\section{Epicardial Echocardiography}

All animals underwent epicardial echocardiography immediately postoperatively, as well as before study end

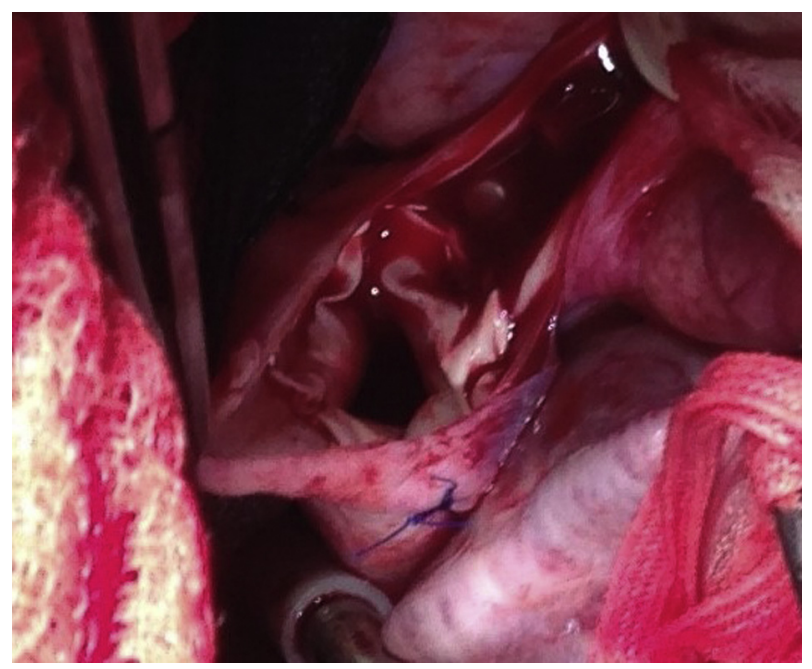

FIGURE 2. Intraoperative photograph of the scaffold-based pulmonary heart valve being sutured in place with 4-0 polypropylene suture after excision of native pulmonary valve. 
points of $1,4,8$, and 12 hours to confirm valve function. The first study animal was successfully extubated but developed noncardiac respiratory complications that required medical management until the planned 8-hour end point. The remaining animals were ventilated through study end points of 1 hour, 4 hours, and 12 hours, respectively. The animal with the previously undiagnosed tricuspid dysplasia showed free leaflet motion and no valvular regurgitation on $1 \mathrm{~L} / \mathrm{min}$ of cardiopulmonary bypass flow before euthanasia at the 1hour time point for inability to wean. The remaining 4 animals were weaned completely from bypass before final echocardiographic evaluation. In all cases, free leaflet motion within the TEHV stent was noted universally with 2dimensional echocardiography, as was adequate prosthetic placement in the proximal pulmonary artery (Figure 3, A).
Color flow Doppler indicated no pulmonic regurgitation, with forward nonturbulent flow through the TEHV (Figure 3, B). Continuous-wave Doppler confirmed forward flow with average peak velocity of $2.0 \pm 0.9 \mathrm{~m} / \mathrm{sec}$. There was no demonstrable regurgitation of the valve on color Doppler examination (Figure 3,C).

\section{Gross Explant Analysis}

All valves were explanted immediately after euthanasia (Figure 4, A). Gross explant analysis showed that the PCUU leaflets were intact, without any signs of structural wear or damage. There was no thrombosis associated with the leaflets (Figure 4, B). The Mg AZ31 stents suffered multiple fracture points because of their brittle nature and normal surgical handling, yet there was no embolization
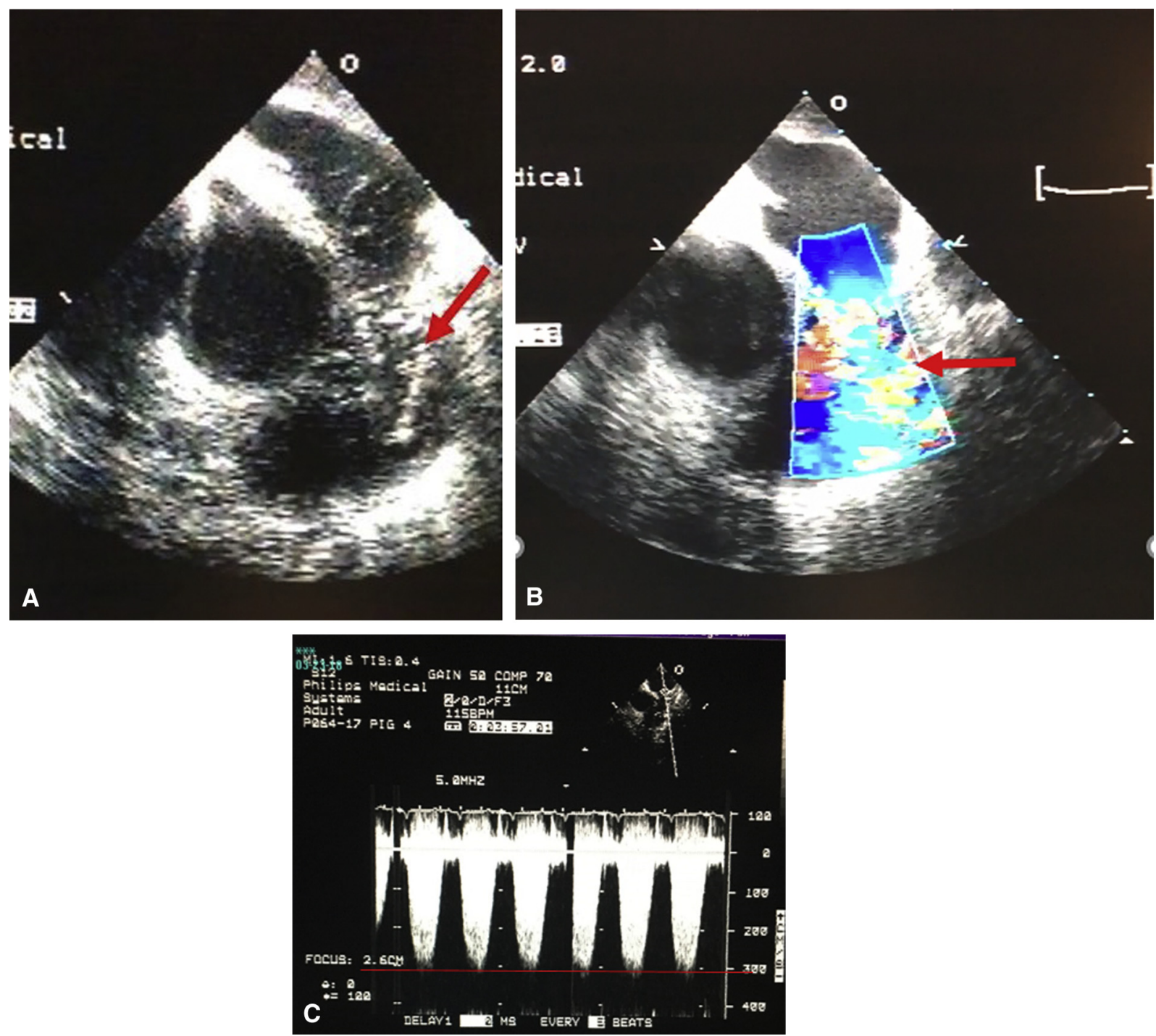

FIGURE 3. A, Two-dimensional epicardial echocardiography after TEHV implant. Red arrow indicates stent housing in the native pulmonary artery. B, Color-flow Doppler shows antegrade flow through the TEHV prosthesis in the pulmonary position; there was no observed regurgitation in this instance. C, Continuous-wave Doppler demonstrating forward flow through the pulmonary prosthesis. 

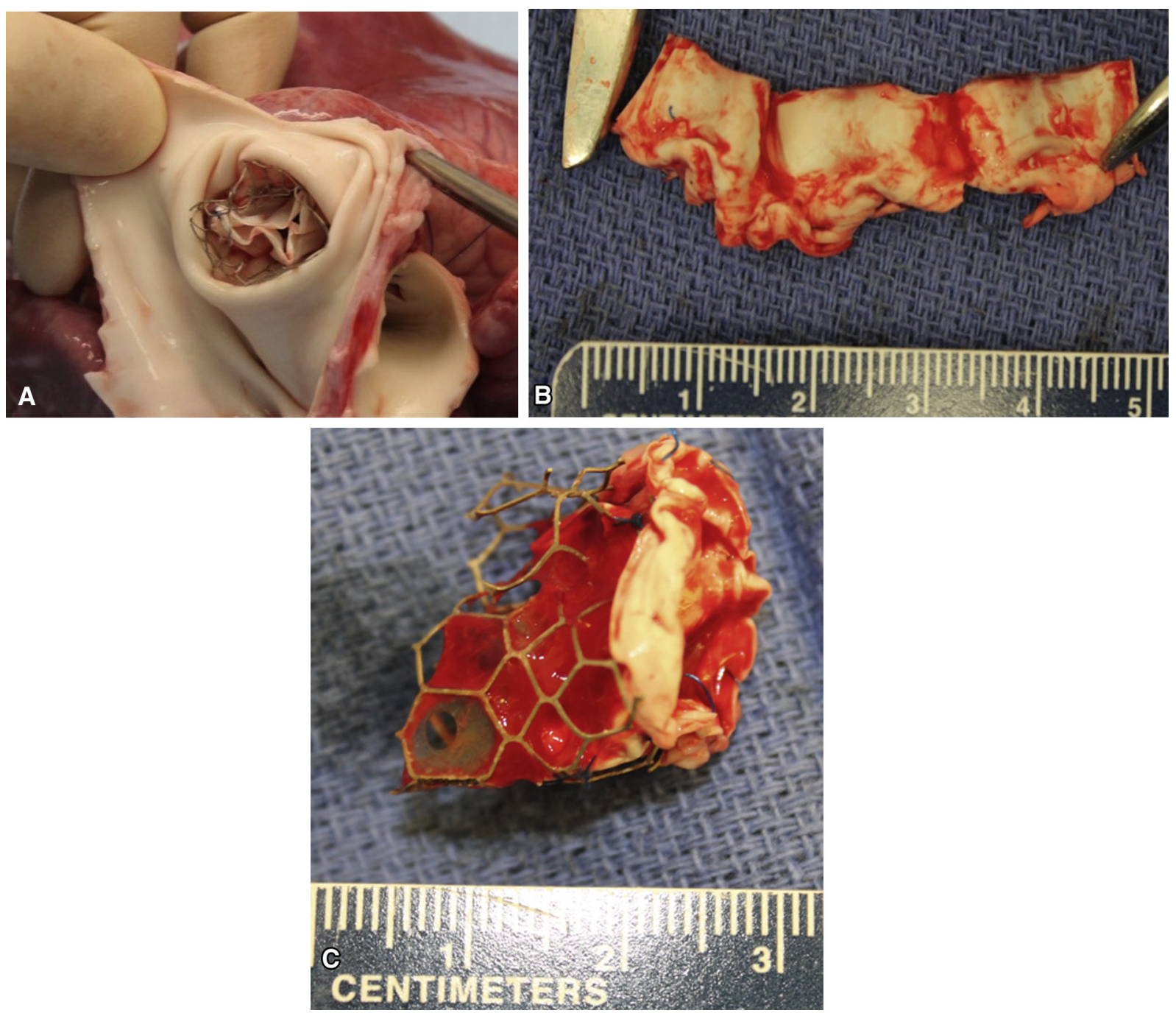

FIGURE 4. A, Gross explant of PCUU pulmonary valve on Mg degradable stent after 12-hour in vivo study. B, PCUU valve leaflets after removal from Mg stent before washing and histologic fixation. No gross thrombosis identified on PCUU leaflets. $\mathrm{C}, \mathrm{Mg}$ stent with fibrin sheath formation after explant after 12-hour in vivo functional assessment. There was no identified obstruction of the valve or outflow tract due to this finding.

noted. On 2 of the 5 stents, there was a distinct nonobstructive nonfilamentous fibrin sheath formed after 6 and 12 hours (Figure 4, C).

\section{Explant Evaluation}

Biaxial stress-strain analysis of explants revealed no difference when compared with the characteristics of the preimplantation sample; this element was consistent with the absence of structural damaged observed by visual inspection. As expected, histologic analysis of the PCUU leaflets up to 12 hours demonstrated no cellular infiltration into the TEHV leaflets and intact PCUU leaflets without evidence of early degradation (Figure 5, A). SEM of the PCUU leaflet demonstrated intact fibrous and porous structure, with no activated platelet deposition or evidence of degradation (Figure 5, B). SEM of the $\mathrm{Mg}$ stent body showed expected early surface oxidation, but no evidence of bulk degradation up to 12 hours (Figure 5,C).

\section{DISCUSSION}

This study demonstrated the acute in vivo function of a novel scaffold-based PCUU TEHV on a degradable $\mathrm{Mg}$ stent frame and confirmed intact histologic and nonthrombogenic ultrastructural integrity with preserved biaxial mechanics. These findings suggest that acute biomechanical function and leaflet biocompatibility are acceptable in the pulmonary position and support chronic animal testing of a biodegradable TEHV.

However, several issues remain to be answered. These include the following: (1) the optimal material and its fabrication to align with natural occurring valvular function and biomechanical properties directed at durability; (2) the histologic biocompatibility to permit endothelialization 

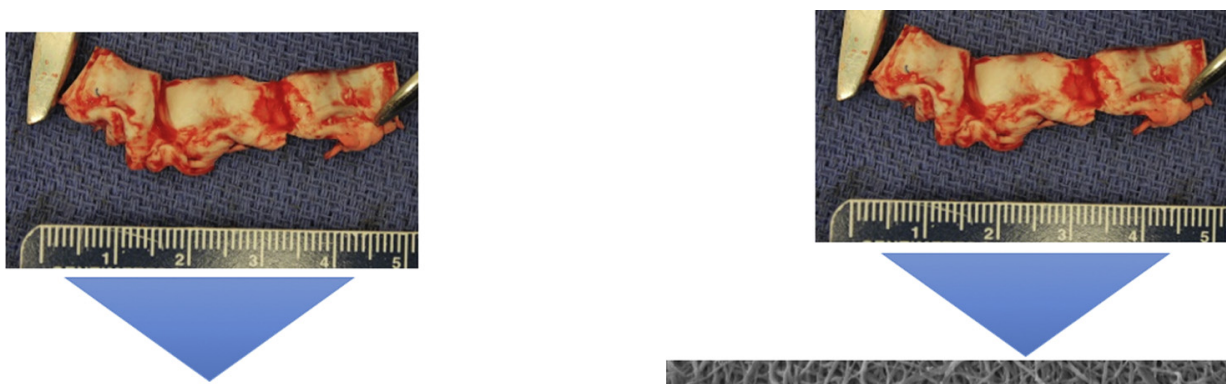

A
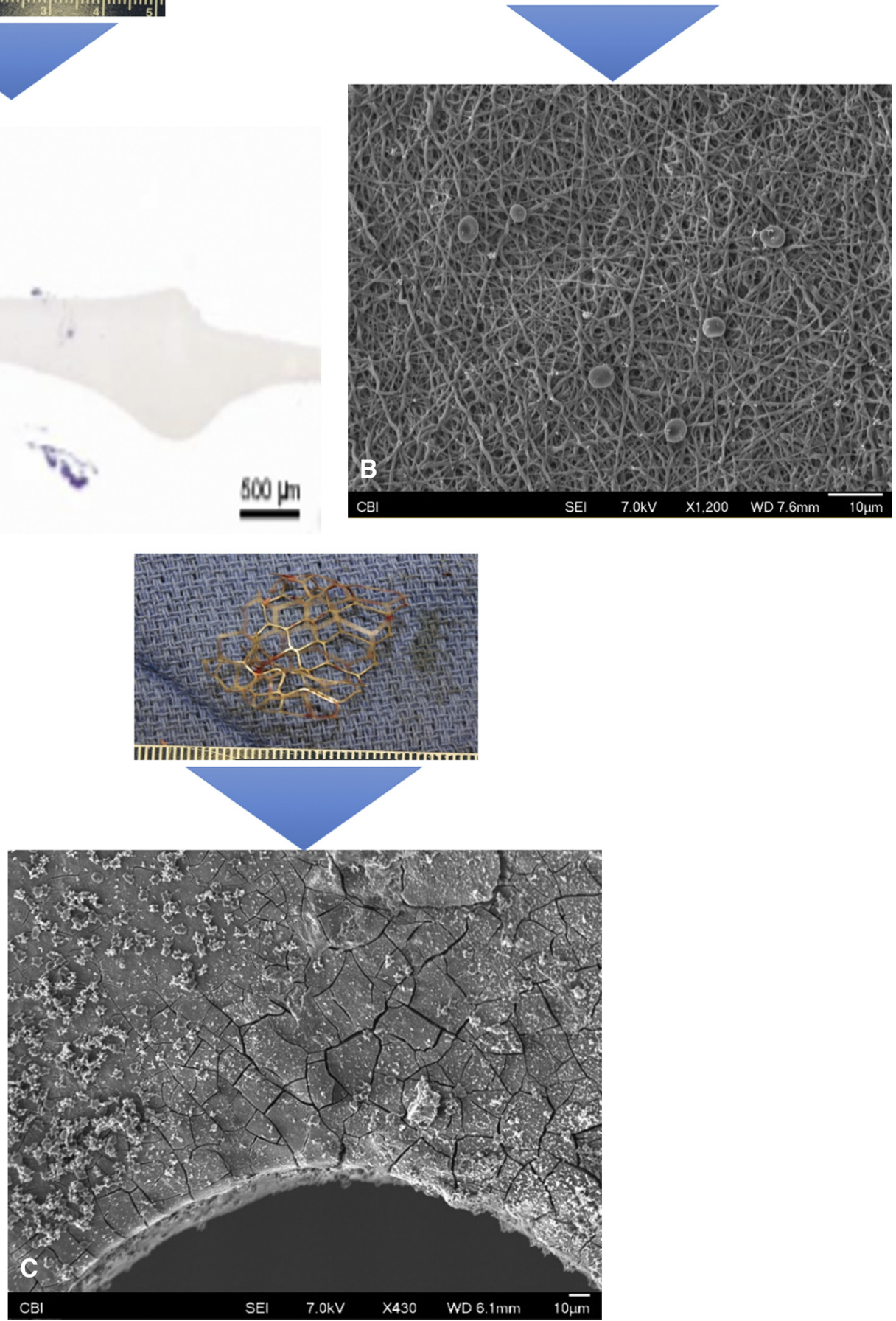

FIGURE 5. A, Hematoxylin-eosin staining of an explanted PCUU valve leaflet. The PCUU leaflet is functionally intact without active cellular uptake. B, PCUU leaflet with SEM shows no change in fibrous/porous structure of the leaflet, and no platelet activation or microthrombus. C, Mg AZ 31 alloy stent shown with SEM displays evidence of early surface oxidation, but no clear bulk degradation. 


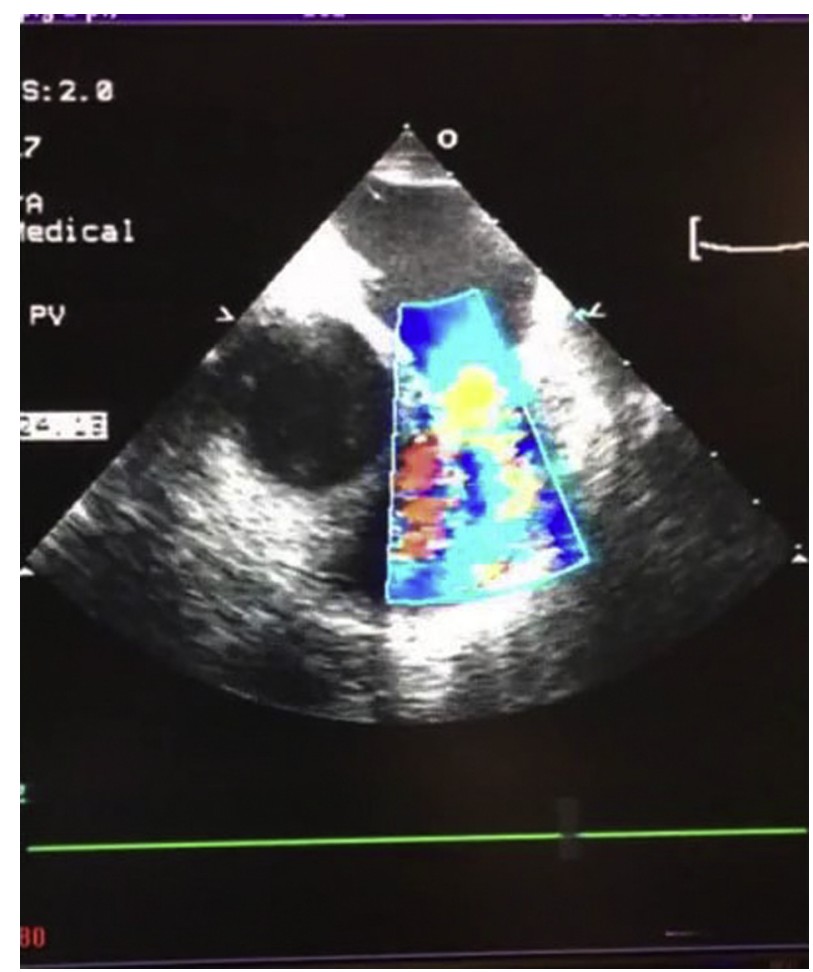

VIDEO 1. Garrett Coyan, MD, MS, describes the fabrication and implantation of a bioinspired scaffold-based engineered heart valve. Adapted from D'Amore and colleagues, ${ }^{9}$ with permission from Elsevier. Video available at: https://www.jtcvs.org/article/S0022-5223(18)33112-X/fulltext.

followed by multilayer valve replication directed at nonthrombogenic functional tissue to permit somatic growth; and (3) the adaptability of the technique of implantation for all positions and the potential for transcatheter application.

The concept of in situ cellularization strategies for TEHVs has been proposed as an alternative to in vitro cellularization strategies. ${ }^{6,12}$ The technique in the current study of DCD electrospinning to fabricate bioinspired PCUU heart valves leverages this paradigm. This preliminary study successfully demonstrated the capability of DCD electrospinning to reproduce a semilunar valve to accurate annular size and leaflet shape configuration. Previous challenges have been to ensure that scaffold creation meets the requirements of a fully functional valve before cell recruitment and replacement with native tissue. ${ }^{7}$ This study of the PCUU TEHV in the pulmonary position represents the first demonstration of this metric for a DCD trileaflet scaffold-based valve system.

An immediately bioavailable and functional scaffoldbased heart valve has potential challenges with short-term functional durability and histologic integrity. This study demonstrated early successful function of the PCUU TEHV in the pulmonary position via epicardial echocardiography. The acute nature of this study did not allow sufficient time for endothelization of the PCUU leaflets, as shown by histology. However, it is important to note that there was no macro or micro thrombosis of the leaflets and no platelet aggregation or inflammatory reaction via SEM up to the 12hour end point. This demonstrates encouraging early leaflet biocompatibility. Further studies will need to include histochemical analysis to identify local inflammatory response, endothelial cell recruitment, stent integration in the wall, calcification, and in situ degradation profile of the polymer. These steps will be critical to identify the need for structural support duration of any biodegradable stent, such as the $\mathrm{Mg}$ alloy stent used in this study. Additionally, the looming questions will be the progressive functionality of the valve during this process as previous studies using TEHV leaflets demonstrate shrinkage that leads to valve failure. ${ }^{13}$ The polyurethane chemistry used to generate the scaffold can readily be manipulated to control its degradation time as in vivo experience might dictate. ${ }^{11,14}$

Implantation of a TEHV may be performed using current techniques for homograft implantation or stentless prostheses. However, providing a biodegradable stent frame that permits functional ingrowth while delivering both ease of implant and the potential for transcatheter application may provide some advantages. ${ }^{15}$ The current study used a $\mathrm{Mg}$ AZ31 alloy stent providing colinear integrity adjacent to the root and commissures in the pulmonary or aortic position that resorbs over time as the valve potentially recruits native tissue for a support system. For this to be successful, clear degradation rates and conditions of both the individual polymeric scaffold and the Mg alloy stent need to be established. ${ }^{14}$ This is currently under study and will add greatly to the potential to combine these 2 technologies. The rate of Mg AZ31 degradation may be too rapid and thus not provide support for a time window long enough to allow for sufficient endogenous tissue growth and mechanical stability of the device. Previous work has established that dip-coating of $\mathrm{Mg}$ stents with biodegradable polyurethanes can improve control and prolong this process, making it tailorable to TEHV leaflet degradation as applied in the current study. ${ }^{11}$ Therefore, polymeric coating, metallic surface alterations, and profile reduction of the stent design may readily mitigate the early platelet activation and fibrin sheath formation observed in the current study. If proven to be histologically and functionally durable, the combination of a $\mathrm{Mg}$ stent with a PCUU TEHV, both with time-driven degradation properties through fabrication, may provide a disruptive valve solution for both congenital and adult application (Video 1).

\section{Study Limitations}

There are several limitations to this study that bear consideration. As a pilot study, the small number of animals and the acute nature of the study limit the ability to make conclusions about the total performance profile of a completely biodegradable TEHV. Additional information from chronic animal 
examinations may further elucidate in vivo functionality. All experiments ended within 12 hours of study initiation, and as reported by several groups, the management of the larger swine postcardiopulmonary bypass can be difficult. Although the PCUU TEHV showed promising functional parameters via echocardiography, the fact that early fibrin sheath formation occurred on the $\mathrm{Mg}$ alloy stent and the stent itself was difficult to handle during the surgery indicates that further development will have to occur to optimize the biodegradable $\mathrm{Mg}$ stent system to work in conjunction with the PCUU TEHV system. Additionally, because of the time constraints of the study, cellularization/endothelization was not able to be observed on the PCUU leaflets. We have observed cellularization of the polyurethane scaffolds in other experimental locations in vivo including as an epicardial patch as well as in abdominal wall replacement, but this will surely need to be readdressed with a chronic valve study focusing on biomechanics of the leaflets, polymer degradation, and cellularization over time. ${ }^{15,16}$ The large swine model may be a limitation for chronic examination because of complications associated with cardiopulmonary bypass, as well as accelerated growth rates and large body weight to cardiac weight ratios. An ovine model may be preferential for longer-term studies of the biodegradable TEHV platform.

\section{CONCLUSIONS}

This study shows that a novel biodegradable scaffoldbased TEHV supported with a biodegradable $\mathrm{Mg}$ alloy stent functions well in an acute in vivo environment. PCUU leaflet mechanics and structure are maintained with up to 12 hours of in vivo blood contact and physiologic stress without valvular thrombus formation. The $\mathrm{Mg}$ stent has evidence of rapid surface oxidation and fibrin sheath formation, which will require additional development before use. This study provides a foundation for chronic studies to examine the degradation profile and in vivo tissue recruitment activity of polymeric scaffold-based heart valves.

\section{Webcast}

You can watch a Webcast of this AATS meeting presentation by going to: https://aats.blob.core.windows.net/media/ 18Apr30/Theater $\% 201 \% 20$ Booth $\%$ 20134/S61\%20-\%20 Annual\%20C.\%20Walton/S61_5_webcast_012955797.mp4.

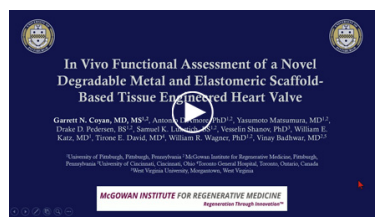

\section{Conflict of Interest Statement}

Authors have nothing to disclose with regard to commercial support.

The authors thank Joseph Hanke, Meegan Ambrose, and the entire staff at the McGowan Center for Preclinical Studies for their assistance with animal care throughout this study.

\section{References}

1. Grunkemeier GL, Furnary AP, Wu Y, Wang L, Starr A. Durability of pericardial versus porcine bioprosthetic heart valves. J Thorac Cardiovasc Surg. 2012;144: 1381-6.

2. Mao J, Wang Y, Philippe E, Cianciulli T, Vesely I, How D, et al. Microstructural alterations owing to handling of bovine pericardium to manufacture bioprosthetic heart valves: a potential risk for cusp dehiscence. Morphologie. 2017;101:77-87.

3. Alavi SH, Ruiz V, Krasieva T, Botvinick EL, Kheradvar A. Characterizing the collagen fiber orientation in pericardial leaflets under mechanical loading conditions. Ann Biomed Eng. 2013;41:547-61.

4. Iung B, Vahanian A. Epidemiology of valvular heart disease in the adult. Nat Rev Cardiol. 2011;8:162-72.

5. Shinoka T, Miyachi H. Current status of tissue engineering heart valve. World J Pediatr Congenit Heart Surg. 2016;7:677-84.

6. Motta SE, Lintas V, Fioretta ES, Hoerstrup SP, Emmert MY. Off-the-shelf tissue engineered heart valves for in situ regeneration: current state, challenges and future directions. Expert Rev Med Devices. 2018;15:35-45.

7. Fan R, Bayoumi AS, Chen P, Hobson CM, Wagner WR, Mayer JE Jr, et al. Optimal elastomeric scaffold leaflet shape for pulmonary heart valve leaflet replacement. J Biomech. 2013;46:662-9.

8. Hobson CM, Amoroso NJ, Amini R, Ungchusri E, Hong Y, D'Amore A, et al. Fabrication of elastomeric scaffolds with curvilinear fibrous structures for heart valve leaflet engineering. J Biomed Mater Res A. 2015;103:3101-6.

9. D'Amore A, Luketich SK, Raffa GM, Olia S, Menallo G, Mazzola A, et al. Heart valve scaffold fabrication: bioinspired control of macro-scale morphology, mechanics and micro-structure. Biomaterials. 2018;150:25-37.

10. Koo Y, Tiasha T, Shanov VN, Yun Y. Expandable Mg-based helical stent assessment using static, dynamic, and porcine ex vivo models. Sci Rep. 2017;7:1173.

11. Gu X, Mao Z, Ye SH, Koo Y, Yun Y, Tiasha TR, et al. Biodegradable, elastomeric coatings with controlled anti-proliferative agent release for magnesium-based cardiovascular stents. Colloids Surf B Biointerfaces. 2016;144:170-9.

12. Hasan A, Saliba J, Pezeshgi Modarres H, Hobson CM, Wagner WR, Mayer JE Jr, et al. Micro and nanotechnologies in heart valve tissue engineering. Biomaterials. 2016;103:278-92.

13. Arsalan M, Walther T. Durability of prostheses for transcatheter aortic valve implantation. Nat Rev Cardiol. 2016;13:360-7.

14. Hashizume R, Hong Y, Takanari K, Fujimoto KL, Tobita K, Wagner WR. The effect of polymer degradation time on functional outcomes of temporary elastic patch support in ischemic cardiomyopathy. Biomaterials. 2013;34: 7353-63.

15. D'Amore A, Yoshizumi T, Luketich SK, Wolf MT, Gu X, Cammarata M, et al. Bi-layered polyurethane - extracellular matrix cardiac patch improves ischemic ventricular wall remodeling in a rat model. Biomaterials. 2016;107:1-14.

16. Takanari K, Hashizume R, Hong Y, Amoroso NJ, Yoshizumi T, Gharaibeh B, et al. Skeletal muscle derived stem cells microintegrated into a biodegradable elastomer for reconstruction of the abdominal wall. Biomaterials. 2017;113: $31-41$.

Key Words: heart valve, tissue engineering, cell regeneration 